\title{
Emergent Diagnosis via Coalition Formation
}

\author{
Carine Webber ${ }^{\prime}$ and Sylvie Pesty \\ Laboratoire Leibniz - IMAG \\ 46, avenue Felix Viallet \\ 38031 Grenoble Cedex France \\ \{Carine.Webber, Sylvie.Pesty\} @imag.fr \\ http://www-leibniz.imag.fr/
}

\begin{abstract}
This paper presents a mechanism of coalition formation where agents solve a problem of diagnosis. Our approach considers that a diagnosis may be seen as the result of an emergent process where findings (at a microscopic level) are interpreted by entities (at a macroscopic level). At the micro-level agents interact and form coalitions. At the macro-level specialized agents are able to interpret coalition formation and infer a diagnosis. Our domain of application is student modelling and in this framework we conceive it as a diagnosis task where a 'state of conceptions' is ascribed to a student based on his/her problemsolving activities.
\end{abstract}

\section{Introduction}

According to Maxion, diagnosis is a form of high-level pattern recognition of symptoms or symbols and it may be one emergent property of certain complex systems [6]. Usually diagnosis is defined as a process of identifying a situation or a system condition from its intrinsic characteristics. Additionally, a diagnosis may allow a system to adapt itself to the immediate constraints of the environment, to resources reallocation, and to the different categories of users.

Essentially, we assume that a diagnosis is a process where microscopic observable findings are recognised by macroscopic entities and they may determine global system's behaviour. For instance, in the case of user-adapted applications, a limited set of elements observed from user interactions may permit a system to diagnose the user's level of expertise and adjust its behaviour according to it. In this case, the level of expertise ascribed to a user could be the result of a diagnosis process.

Systems having multiple interacting components and a behaviour that cannot be simply inferred from the behaviour of the components are qualified as complex [9]. Diagnosis, as a product, may be an emergent property of some complex systems. Multi-agent approach brings some advantages for modelling complex systems since: (1) its application is not dependent on the number of agents (contrarily to certain approaches where a large number of elements is necessary); (2) agents can have heterogeneous behaviours; (3) interactions of different levels of complexity are allowed; (4)

\footnotetext{
* Scholar of Capes/Cofecub, Brazil.

F.J. Garijo, J.C. Riquelme, and M. Toro (Eds.): IBERAMIA 2002, LNAI 2527, pp. 755-764, 2002.

(C) Springer-Verlag Berlin Heidelberg 2002
} 
it is applicable to several domains (social sciences simulations, computational economy, ecology, physics, and so on).

The idea that systems constituted of several agents having simple behaviour (a behaviour described by a few rules, for instance) can show a dynamic global behaviour having properties not easily predictable (even if the external condition are known) is exploited in this paper. A few examples have demonstrated such emergent and unpredictable behaviour. For instance, simulations in the domain of voting theory have shown how parties emerge from voters' choices [8,11]. Also, experiments in computational economics have illustrated the emergence of markets based on the behaviour of agents representing costumers and vendors [12] as well as the emergence of social classes and social norms [1]. In a similar fashion, the two domains apply coalition formation mechanisms to simulate and study social behaviours. But beyond simulation purposes, coalitions are as well applied to problem solving. Task allocation has been one of the most applicable examples of coalition formation [10]. Most recently, electronic marketplace has shown to enclose enough dynamic aspects to constitute an excellent testbed for mechanisms of coalition formation.

The work we describe here considers diagnosis as a problem-solving task and we propose to solve it by coalition formation. In addition, we assume that user modelling, more specifically student modelling, is a process of diagnosing the 'state of conceptions' hold by an student in interaction with a learning environment [13]. The diagnosed conceptions are ascribed to the student and kept in his/her student model to guide pedagogical decisions (the choice of problems, advices, etc.) of Baghera, a distance learning environment [13]. The framework we propose for student modelling is based on the model of conceptions [2], developed in mathematics education.

This paper is organised as follows. Next section briefly describes the theoretical framework of our approach. Third section introduces the multi-agent systems for diagnosing conceptions. Fourth section describes the elements composing the system and mechanisms for coalition formation. Finally, some experiments realised are described.

\section{Theoretical Framework}

\subsection{Emergence}

An emergent system is characterised by having a behaviour that cannot be predicted from a centralised and complete description of the component units of the system [5]. In emergent systems, the overall behaviour is the result of a great number of interactions of agents obeying very simple laws. The overall behaviour cannot be anticipated by simple reduction to individual behaviours, following a logico-deductive model, but it is rather conditioned by the immediate surroundings, like other agents and objects in the environment.

Very often the definition of emergence is attached to the notion of levels and detection [3]. For this reason diagnosis can be effectively seen as an emergent property of certain complex systems, since in a diagnosis process lower-level symptoms or 
symbols are recognised by higher-level entities [6]. Note that emergent objects have a representation distributed over many different elements. Each of these elements may take part of many different objects simultaneously. This may be observed in the classifier systems proposed by Forrest [5], in the system for diagnosis of communication networks [6], and in the emergence of conceptions discussed in this paper.

\subsection{Conception Theory}

Conception theory was developed in mathematics education with a cognitive and didactical foundation [2]. In this model a conception is characterised by a quadruplet $\mathrm{C}$ $(\mathrm{P}, \mathrm{R}, \mathrm{L}, \Sigma)$ where:

$-P$ represents a set of problems;

$-\mathrm{R}$ represents a set of operators involved in the solutions of problems from $\mathrm{P}$;

$-\mathrm{L}$ is a representation system allowing the representation of $\mathrm{P}$ and $\mathrm{R}$;

$-\Sigma$ is a control structure (details are given at section 3.1).

An element from any set can contribute to the characterisation of several different conceptions; for example two conceptions may share problems in their domain of validity or may have common operators. For the sake of brevity it is not possible to give in this paper more details about this theory. However, we propose the examples presented in figures 1 and 2.

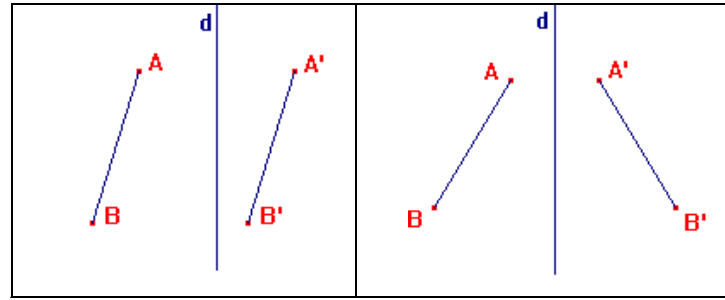

Fig. 1. Parallelism

Fig. 2. Reflection
Figure 1 presents a construction made by a student holding a misconception stating that "if two line segments are symmetrical then they are parallel". Figure 2 was constructed by a student holding the correct conception of reflection.

In order to illustrate how diagnoses occur, we examine the problem described on figure 3 .

\footnotetext{
Problem statement: Let $\mathrm{ABC}$ be an equilateral triangle. $A^{\prime}$ is symmetrical to A with respect to line $d$. $L$ is the middle point of $[A B], M$ is the middle point of $[\mathrm{BC}]$, and $\mathrm{N}$ is the middle point of $[\mathrm{AC}] . \mathrm{P}$ is the intersection point of lines (LM) and (CA'). $\mathrm{O}$ is the intersection point of lines (NM) and (BA'). What is the symmetrical line segment of [NM] with respect to line d? Construct your proof.
}

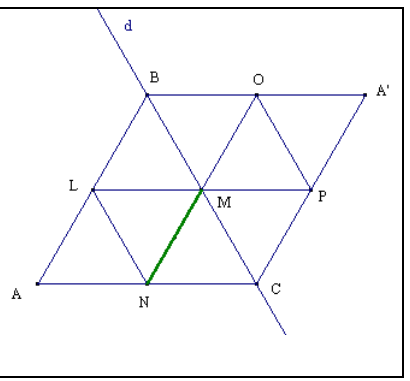

Fig. 3. A problem in the domain of reflection 
In this problem students are asked to prove, using geometrical properties of reflection, that the line segment $[\mathrm{NM}]$ has a symmetrical object with respect to the axis $d$. Let's consider one strategy to solve it, which consists on proving each step from table 1. In the case of step 6, we consider four alternatives (6.a, 6.b, 6.c and 6.d) in order to exemplify how students holding different conceptions would express the solution. First, consider the proof composed by steps 1-2-3-4-5-6.a where the student has proven that $[\mathrm{OM}]$ is the symmetrical line segment of $[\mathrm{NM}]$. This solution, by the operators used to construct it (6.a1-6.a7), characterizes the so-called misconception of 'central symmetry'.

Table 1. Possible strategies to solve the problem

\begin{tabular}{|c|c|c|}
\hline 1 & & $\mathrm{~A}^{\prime} \mathrm{BC}$ is an equilateral triangle \\
\hline 2 & & $\mathrm{ABA}^{\prime} \mathrm{C}$ is a lozenge $\left([\mathrm{AB}] / /\left[\mathrm{CA}^{\prime}\right]\right.$ and $\left.\left[\mathrm{BA}^{\prime}\right] / /[\mathrm{AC}]\right)$ \\
\hline 3 & & {$[\mathrm{AB}] / /\left[\mathrm{CA}^{\prime}\right] ;[\mathrm{AB}] / /[\mathrm{NO}] ;[\mathrm{NO}] / /\left[\mathrm{CA}^{\prime}\right]$} \\
\hline 4 & & $\mathrm{O}$ is the middle point of $\left[\mathrm{A}^{\prime} \mathrm{B}\right]$ \\
\hline 5 & & $\mathrm{P}$ is the middle point of $\left[\mathrm{A}^{\prime} \mathrm{C}\right]$ \\
\hline \multirow[t]{7}{*}{$6 . \mathrm{a}$} & 1 & M is its own symmetrical point with respect to $d$ \\
\hline & 2 & As $[\mathrm{AC}] / /\left[\mathrm{BA}^{\prime}\right]$ and \\
\hline & 3 & $\mathrm{~N}$ is the middle point of $[\mathrm{AC}]$ and \\
\hline & 4 & $\mathrm{O}$ is the middle point of $\left[\mathrm{A}^{\prime} \mathrm{B}\right]$ and \\
\hline & 5 & Line segments $[\mathrm{NM}]$ and $[\mathrm{OM}]$ have the same size \\
\hline & 6 & $\mathrm{O}$ is the symmetrical point of $\mathrm{N}$ \\
\hline & 7 & So, $[\mathrm{OM}]$ is the symmetrical line segment of $[\mathrm{NM}]$ with respect to point $\mathrm{M}$ \\
\hline \multirow[t]{5}{*}{ 6.b } & 1 & $\mathrm{M}$ is its own symmetrical point with respect to $\mathrm{d}$ \\
\hline & 2 & Line segments $[\mathrm{NM}]$ and $[\mathrm{PM}]$ have the same size \\
\hline & 3 & {$[\mathrm{NP}]$ is perpendicular to axis $\mathrm{d}$} \\
\hline & 4 & $\mathrm{P}$ is the symmetrical point of $\mathrm{N}$ \\
\hline & 5 & So, $[\mathrm{PM}]$ is the symmetrical line segment of $[\mathrm{NM}]$ with respect to $\mathrm{d}$ \\
\hline \multirow[t]{5}{*}{ 6.c } & 1 & As $[\mathrm{NO}] / /\left[\mathrm{CA}^{\prime}\right]$ and $[\mathrm{NM}] / /\left[\mathrm{PA}^{\prime}\right]$ \\
\hline & 2 & As $[\mathrm{NM}]$ and $\left[\mathrm{PA}^{\prime}\right]$ are parallels and have the same size, they are symmetrical. \\
\hline & 3 & $\mathrm{P}$ is the symmetrical point of $\mathrm{N}$ \\
\hline & 4 & $\mathrm{~A}^{\prime}$ is the symmetrical point of $\mathrm{M}$ \\
\hline & 5 & So, $\left[\mathrm{PA}^{\prime}\right]$ is the symmetrical line segment of $[\mathrm{NM}]$ with respect to $\mathrm{d}$ \\
\hline \multirow[t]{5}{*}{$6 . d$} & 1 & As $[\mathrm{NO}] / /\left[\mathrm{CA}^{\prime}\right]$ and $[\mathrm{NM}] / /[\mathrm{CP}]$ \\
\hline & 2 & As $[\mathrm{NM}]$ and $[\mathrm{CP}]$ are parallels and have the same size, they are symmetrical. \\
\hline & 3 & $\mathrm{P}$ is the symmetrical point of $\mathrm{N}$ \\
\hline & 4 & $\mathrm{~A}^{\prime}$ is the symmetrical point of $\mathrm{M}$ \\
\hline & 5 & So, $[\mathrm{CP}]$ is the symmetrical line segment of $[\mathrm{NM}]$ with respect to $\mathrm{d}$ \\
\hline
\end{tabular}

The second alternative (1-2-3-4-5-6.b) gives the correct answer ([PM] is the symmetrical segment of $[\mathrm{NM}])$ and its attached to the conception of reflection. Third (1-23-4-5-6.c) and forth (1-2-3-4-5-6.d) alternatives, even though they give different answers, they characterize the same misconception of 'parallelism'. In these cases (6.c and 6.d), students state that two line segments are symmetrical if they are parallel and have the same size and it is possibly an inversion of the correct operator, which states that two parallel line segments having the same size are symmetrical with respect to a parallel axis.

It is important to note that a diagnosis is based on a sequence of problems solved by the student. Different problems in a well-oriented sequence permit the construction of 
a student model having enough information to characterize student's conceptions in a specific domain of knowledge [13].

\subsection{Voting Theory}

Voting models are widely used in social sciences and have their roots in Game Theory. Social sciences research about voting has been investigating new approaches to studying voting schemes, voter behaviour, and the influences of manipulation of votes and insincere voting. Studies based on simulation of elections have led to models providing explanations to voter behaviour, so as explanations to group decisions and coalition formation.

In the domain of multi-agent systems, voting theory has been used as a technique for reaching consensus in a negotiation process and group-decision making [7]. In the simulation of coalitions formation, agents have been used to demonstrate how it occurs from individual voter preferences [8].

Furthermore, it has been shown that emergent structures can be resultant of a voting process. Schreiber [8] has demonstrated through multi-agent simulations that elites and parties are emergent consequences of the behaviour and preferences of voters.

In essence our interest in voting theory relies on the possibility of capturing group decision as well as modelling the influence of an agent preference over the preferences of the rest of agents.

Our approach is based on spatial models of simulation of voting behaviour. Spatial voting theory has its origins in the field of Political Science [11]. This model assumes that political issues can be quantified and therefore voters and candidates can be represented by points in a so-called issue space. Similarly, each candidate is described by a platform position in the issue space.

Usually this space is viewed as the Euclidian vector space $\mathrm{R}^{\mathrm{I}}$ having I dimensions. Each voter is represented by a vector in the issue space, called ideal point that represents its opinion on each issue. In these models, voters form coalitions with other voters close to them in the issue space. Coalitions start with a small number of voters and possibly form coalitions with other coalitions to increase their potential. A hierarchy of coalitions is built until a coalition is created with the majority of voters. The coalition with the majority rules and the competing coalitions adapt platforms to gain greater support. Each voter may represent a single voter or a team of voting people. Agents may be voters or coalitions and, in the last case, they may represent an aggregation of voters but having no votes by themselves. The action in this model takes place only in the issue space.

\section{Multi-agent Architecture}

We have employed the AEIO methodology [4] for the multi-agent-oriented analysis and design of our diagnosis system. This methodology considers the problem to be modelled as composed by four elements: Agents, Environment, Interactions and Or- 
ganization. The first step of the methodology consists in defining the four elements and then they can be formalised.

We propose a multi-agent system where, at a microscopic level, agents behave and interact and, at a macroscopic level, coalitions emerge. The emergence of coalitions is interpreted as the emergence of a diagnosis of student's conceptions.

The conception theory allows to model student's conceptions. However, conceptions are not elements possible to be directly observed. Observable elements are operators used by student, the problem solved, the language used to express them, and theoretical control structures. For this reason, the micro-level is composed by elements (from the quadruplet) defining a conception. At the macro-level, conceptions can be seen as sets of agents of four categories: problems, operators, language and control. Each element from the quadruplet is the core of one particular agent.

\subsection{Agents}

An agent, in a given time slot, can be either active or inactive. This state can vary according to changes it perceives in its environment. The first action of any agent is to check whether the element it represents is present in the environment. In the presence of the element, the agent becomes satisfied. Once satisfied, the agent takes part in the issue space and it is able to form coalitions. Notice that an agent knows previously to which conceptions the element it represents belongs. A description of the role of each category of agents is given below.

Problem Agents. A problem agent becomes satisfied when the category of problems it represents is present in the environment. In the domain of reflection, a category of problems is described by four didactical variables named: line of symmetry orientation, segment orientation, angle formed between line of symmetry and line segment and intersection formed between the line of symmetry and line segment. The combination of the different values that these didactical variables could take, leads to problems of different complexity, allowing to focus on different aspects of the learning of reflection and most important, allowing the expression of different conceptions.

Operator Agents. An operator agent becomes satisfied when the element $r$ of $R$ it represents, is present in the solution constructed by the student. An operator transforms a problem in a new problem. A sequence of operators leads to the problem solution. An example of an operator is as follows: if two symmetrical objects have one point in common, then this point belongs to the axis of symmetry.

Language Agents. A language agent becomes satisfied when the element 1 of $\mathrm{L}$ it represents, is present in the solution constructed by the student. It can be a grammar, a graphical representation, or an alternative way of expression allowing the description of the problem and the solution. Given that our problems ask for the construction of a proof, the language is based on a grammar (for reasons of brevity it is not presented here).

Control Agents. A control agent becomes satisfied when the element s of $\Sigma$ it represents, is present in the solution constructed by the student. During problem solving, learners choose operators, validate actions and validate the final result. Each of these three decisions is guided by control structures. Control elements are perceptive when 
attached to the fact that the learner makes assertions based on something "seen" on the screen and uses this information to take and validate decisions. On the other hand, control structures are theoretical when a learner bases decisions and validations on knowledge previously acquired. Reflection involves many visual elements of control; for instance, a learner holding the conception of parallelism may accept that a problem is correctly solved when the image line segment "looks" parallel to the original line segment. In the case of our system, we consider only theoretical controls and some perceptive controls that can be expressed by means of a proof.

\subsection{Environment}

The environment represents the external world that agents have to deal with and it coevolves with the agents. In the environment there is a representation of the problem solved by the student, the proof corresponding to the student's solution and the issue space where coalition formation takes place.

\subsection{Interactions}

Agents interact with the environment and through these interactions they transform the environment (issue space). Such transformation generates changes in agents' behaviours. The cycle of interactions continues indefinitely until no more coalitions can be formed or merged.

\subsection{Organisations}

We apply a dynamic approach to conceive organisations. We consider that agents form dynamically coalitions when they are needed to solve a problem. Our approach of coalitions formation is based on emergent approach. Moreover, it is considered that once the problem is solved, coalitions are not applicable anymore for a later processing.

In the next section we proceed with the formalisation of the most relevant components of the model.

\section{Formal Description}

\subsection{Environment}

The environment is described by a set $E n v=\{P R, S P, I S\}$ where PR represents the problem that the student has solved, SP represents the proof constructed by the student as a solution to the problem PR and IS is the issue space. The space of votes $\mathbb{R}^{\mathrm{I}}$ is the Euclidian space having I dimensions. The number of conceptions to be diagnosed determines the number of space dimensions. Voters are represented by a position in the space corresponding to their 'opinions' about candidate conceptions. A position is represented by a vector $\mathrm{v} \in \mathbb{R}^{\mathrm{I}}$. 


\subsection{Diagnosis Problem}

Given a set of conceptions $C=\left\{c_{1}, \ldots, c_{i}\right\}$, a set of $n$ agents $A=\left\{a_{1}, \ldots, a_{n}\right\}$ and a set representing the state of the environment $E n v=\{P R, S P, I S\}$, the problem we propose to solve consists in assigning one or more (possibly concurrent) groups $G$ of agents $(G$ $\subset A)$ representing the state of student's conceptions reflected in the environment.

\subsection{Agent}

Let $C$ be a set of conceptions $\left\{C_{1}, C_{2}, \ldots, C_{n}\right\}$. Consider that any conception $C_{i}$ in $C$ is defined by a quadruplet $\left(\mathrm{P}_{\mathrm{i}}, \mathrm{R}_{\mathrm{i}}, \mathrm{L}_{\mathrm{i}}, \Sigma_{\mathrm{i}}\right)$ where :

- $P_{i}$ is a set of problems $\left\{P_{1}, P_{2}, \ldots, P_{n}\right\}$ of $C_{i}$;

- $R_{i}$ is a set of operators $\left\{r_{1} r_{2}, \ldots, r_{n}\right\}$ of $C_{i}$;

- $L_{i}$ is a grammar for the expression of $P_{i}$ and $R_{i}$ of $C_{i}$;

- $\Sigma_{\mathrm{i}}$ is a set of control structures $\left\{\sigma_{1}, \sigma_{2}, \ldots, \sigma_{n}\right\}$ of $c_{i}$.

Let $A$ be a set of agents $\left\{a_{1}, \ldots, a_{n}\right\}$. Let $K_{i}$ be a set of $n$ candidate conceptions $\left\{K_{11}\right.$, $\left.k_{i 2,} \ldots, k_{i n}\right\}$ for an agent $a_{i}$ where $K_{i} \subset C$. Let $E$ be a set of elements $\left\{e_{1, \ldots}, e_{n}\right\}$ from the conceptions formalisation and assume that $e_{i}$ is the element in the core of an agent $a_{i}$. About any element $e_{i}$ it is known that $e_{i} \in P_{i}\left|e_{i} \in R_{i}\right| e_{i} \in L_{i} \mid e_{i} \in \Sigma_{i}$. Let $Q_{i}$ be the set of acquaintances of an agent $\mathrm{a}_{\mathrm{i}}$ (acquaintances are detailed later in this paper). Finally, $\mathrm{V}$ is the set of votes $\left\{\mathrm{V}_{1 \mathrm{k},}, \ldots, \mathrm{V}_{\mathrm{ik}}\right\}$ given by the agent to preferred candidate conception from $K_{i}$. An agent $a_{i}$ is defined by : an identifier $N_{i}$, an internal state $S_{i} \in$ \{ satisfied, unsatisfied\}, a set of acquaintances $Q_{i}$ a set of candidates conceptions $K_{i}$, an element $e_{i}$, a satisfaction function $f_{i}\left(e_{i}\right.$, Env) and a vector $V_{i}$ representing its starting position in the Euclidian space $\mathbb{R}^{I}$.

\subsubsection{Agent Behaviour}

Agents are created in an unsatisfied state and the satisfaction function may change its state to a satisfied one. When an agent becomes satisfied, it creates its vector to be added to the issue space. Since it is situated in the issue space, its acquaintances are set (section 4.3.2). Agents start forming coalitions with each member of its acquaintances list. Any agent may take part in any number of coalitions. Once an agent takes part in proposed coalitions, it can accept or refuse it. Besides, an agent tries to merge coalitions in which it takes part into. And finally, agents are free to enter and leave coalitions at any time. When it is not possible anymore for an agent to execute any of these actions, the agent stops. The major steps of the algorithm defining the behaviour of an agent are as follows: (1) initialise the data structure; (2) calculate agent's power of voting; (3) set agent's acquaintances; (4) while (list of proposed coalitions is not empty): (4.1) propose coalitions; (4.2) accept coalitions; (4.3) refuse coalitions; (4.4) calculate coalition's utility; (4.5) merge coalitions; and (4.6) abandon a coalition.

\subsubsection{Finding Acquaintances}

The most important feature of an agent is its voting vector, representing its choice of 
candidate conceptions. Its acquaintances represent a list of agents that are spatially located close to it in the issue space.

Let $a_{\alpha}$ and $a_{\beta}$ be two different agents. Let $V_{\alpha}$ and $V_{\beta}$ be vectors representing respectively the positions of $\mathrm{a}_{\alpha}$ and $\mathrm{a}_{\beta}$ in the space $\mathbb{R}^{\mathrm{I}}$. Let $\mathrm{Q}_{\alpha}$ and $\mathrm{Q}_{\beta}$ be the set of acquaintances (initially empty) of respectively $a_{\alpha}$ and $a_{\beta}$. We assume that $a_{\alpha}$ and $a_{\beta}$ are acquaintances if they satisfy the neighbourhood condition.

Neighbourhood condition is calculated by the formula of Euclidian distance between the two vectors $\mathrm{V}_{\alpha}$ and $\mathrm{V}_{\beta}$. The two agents satisfy the condition if the distance is a value under a specific threshold and in this case, $a_{\beta} \in Q_{\alpha}$ and $a_{\alpha} \in Q_{\beta}$. Otherwise, $a_{\alpha}$ and $a_{\beta}$ are not acquaintances to each other.

\subsection{Coalition Formation}

A coalition is a nonempty subset $C o$ of $A$ and it has as utility value the sum of utilities of all agents belonging to it. We follow the traditional approach of coalition formation in the domain of multi-agent systems [7].

The initial number of coalitions is reduced since the initial coalitions are formed between any two agents situated spatially close in the issue space. When agents form a coalition it has a status of proposed coalition and when it is accepted by all of its members it becomes an accepted coalition.

\section{Experiment and Final Considerations}

We have run a diagnosis considering the proof presented at section 2.2 (steps 1-2-3-45-6c). The multi-agent system is composed of 101 agents, distributed as follows: 60 operator agents, 30 problem agents and 11 control agents. As explained before, agents become active if the element represented is found in the proof. For this experiment, 13 agents (1 problem agent, 10 operator agents and 2 control agents) have become active. The issue space has 4 dimensions, representing 4 conceptions on reflection (central symmetry, oblique symmetry, parallelism and reflection). Vectors of agents' opinions have 1 in each dimension representing a good candidate and 0 otherwise. The threshold for calculating acquaintances was 1 .

In the end of this experiment two coalitions have emerged. The greatest number of coalitions, reached at interaction number 150, was of 134 coalitions. Coalition formation was stopped when this number was reduced to 2 coalitions and no relevant changes in the system were observed. The coalition having the greatest utility is considered the winner. Among the 13 agents involved in the diagnosis process, 10 of them took part of the winner coalition. In this experiment, the winner represents the misconception of parallelism; the second coalition represents the conception of reflection (the correct one). The result is satisfactory and it indicates that the student possibly holds the misconception of parallelism. We interpret the fact that a weak coalition (representing reflection) has appeared as a result of correct operators and properties present in the student's proof; they certainly must have a weight in the diagnosis proc- 
ess. However, incorrect operators have appeared as well and they have induced the diagnosis of parallelism by forming the strongest coalition.

As long as the diagnosis process is over, macro-agents (belonging to Baghera main multi-agent system) take pedagogical decisions based on it. For instance, tutor agents may propose new problems to confirm a diagnosis or propose a problem where incorrect procedures of resolution fail, according to pedagogical strategies.

The main challenge of this work has been to define and implement a computational framework to model student's conception supported by a theory developed in the domain of mathematics education. We start assuming that a diagnosis is a kind of problem-solving task. We have proposed in this paper to solve it by coalition formation. However, other approaches, such as those based on probabilistic models (Bayesian networks, for instance) have been studied and will be the object of new implementations.

\section{References}

1. Axtell, R., Epstein, J., Young, H.P. The emergence of classes in a multi-agent bargaining model. Center on Social and Economics Dynamics, Working paper no.9, February 2000.

2. Balacheff N., Gaudin N. Modelling students conceptions - the case of functions. Mathematical Thinking and Learning - an International Journal, 2002. (to appear).

3. Bonabeau, E.; Dessalles, J.; Grumbach, A. Characterizing Emergent Phenomena (1): A critical review. In: Revue Internationale de Systémique, vol.9, n³, 1995, pp. 327-346.

4. Demazeau, Y. From Interactions to Collective Behaviour in Agent-Based Systems. In: Proceedings of the 1st. European Conference on Cognitive Science. Saint-Malo, France, 1995.

5. Forrest, S. Emergent Computation. Physica D 42, 1990. Elsevier Science Publishers, North-Holland.

6. Maxion, R.A. Toward diagnosis as an emergent behavior in a network ecosystem. In: Physica D 42, 1990, pp. 66-84. Elsevier Science Publishers, North-Holland.

7. Sandholm, T.W. Distributed Rational Decision Making. In: Multiagent Systems: A Modern Introduction to Distributed Artificial Intelligence, Weiß, G., MIT Press, 1999. pp.201258.

8. Schreiber, D. The emergence of parties: an agent-based simulation. In: The Annual Meeting of the Midwestern Political Science Association, Chicago, Illinois, 2000.

9. Schweitzer, F.; Zimmermann, J. Communication and Self-Organisation in Complex Systems: A Basic Approach. In: Knowledge, Complexity and Innovation Systems (Eds. M.M. Fischer, J. Frohlich) Advances in Spatial Sciences. Springer, Berlin 2001, pp.275-296.

10. Shehory, O.; Kraus, S. Task allocation via coalition formation among autonomous agents. In: Proceedings of IJCAI'95, Montreal. pp.655-661.

11. Stadler, B.M.R. Adaptative Platform Dynamics in Multi-Party Spatial Voting. Adv.Complex Systems (1999) vol.2, 101-116.

12. Vriend, N.J. Self-Organization of Markets: An Example of a Computational Approach. Computational Economics, 1995, Vol. 8, No. 3, pp. 205-231.

13. Webber, C.; Pesty, S.; Balacheff, N. A multi-agent and emergent approach to learner modelling. In: ECAI 2002 - Proceedings of the 15th European Conference on Artificial Intelligence. F. van Harmelen (ed.), IOS Press, Amsterdam, 2002. pp.98-102. 